\title{
The influence of antimycobacterial chemotherapy on delayed hypersensitivity skin-test reactions in leprosy patients
}

\author{
I A CREE,* W C S SMITH,* R J W REES $\dagger$ \\ \& J SWANSON BECK* \\ *Departments of Pathology and Community Medicine, Ninewells \\ Hospital and Medical School, Dundee, DDI 9SY, Scotland; \\ $\dagger$ Division of Communicable Diseases, Clinical Research Centre, \\ Harrow, Middlesex HAl 3UJ, England
}

Accepted for publication 10 November 1987

\begin{abstract}
Summary Skin tests using purified protein derivative (PPD) and Rees' skin test antigen (RSTA), a soluble extract of Mycobacterium leprae, were performed in 53 treated leprosy patients, 52 newly diagnosed untreated leprosy patients and 78 household contacts of untreated leprosy patients in northern Bangladesh. In addition, a small group of 20 leprosy hospital workers and a further group of 50 indigenous subjects with no known exposure to leprosy were studied.

Untreated paucibacillary and multibacillary patients showed significantly fewer positive reactions than comparable groups of treated patients to both PPD and RSTA. It appears from these results that treatment of leprosy patients is associated with enhanced ability to produce a delayed-type hypersensitivity response to mycobacterial antigens. The mechanisms underlying this phenomenon may include both general and specific suppression of antimycobacterial delayed-type hypersensitivity. The household contacts and indigenous subjects showed similar skin test responsiveness, but virtually all of the hospital workers responded to both PPD and RSTA. The implications of these results for studies of immunity in leprosy patients are discussed.
\end{abstract}

\section{Introduction}

Treatment of leprosy patients, particularly those with borderline leprosy, often leads to exacerbations of the disease known as reversal reactions which are characterized by neuritis and inflammatory changes in the skin lesions. Untreated patients with nonpolar leprosy are susceptible to downgrading reactions which are accompanied by an increase in the bacillary index. In both types of reaction nerve damage occurs and the prevention or early treatment of such exacerbations is an important aspect of patient management. The clinical signs can be correlated with histological and in vitro immunological changes which suggest alterations in delayed-type hypersensitivity (DTH).$^{1-3}$ However, there has been relatively little investigation in vivo of the relationship between DTH and treatment using skin tests. 
In 1962, Guinto \& Mabalay ${ }^{4}$ performed an extensive study of Mantoux testing in leprosy patients. They showed that lepromatous patients had a lower response rate to PPD than tuberculoid patients or healthy controls. In addition, they noted that tuberculoid cases in reaction, bacteriologically positive, gave a tuberculin rate approximating to that of the lepromatous group, while that of the nonreactional cases resembled that of healthy controls'. Many of these recently admitted patients might now be classified as having borderline leprosy with downgrading reaction, so these findings are in keeping with the results of in vitro studies of DTH. Recently 'New Tuberculins' have become available which contain a higher proportion of specific antigens ${ }^{5,6}$ allowing skin test reactivity to mycobacteria to be ascertained with greater specificity. We have used a new tuberculin derived from $M$. leprae, known as Rees' skin test antigen (RSTA) or Leprosin A (Batch CD19), to test reactivity to M. leprae. The standard Mantoux test was also performed since purified protein derivative (PPD) of $M$. tuberculosis contains a large proportion of common mycobacterial antigens in addition to $M$. tuberculosis specific antigen.

\section{Materials and methods}

\section{SUBJECTS}

The study was performed at the HEED Leprosy Hospital, in the Sylhet region of northern Bangladesh, during the first 3 months of 1986. Untreated patients were obtained during population survey or presented to the local control team as self-referrals: treated patients were hospital inpatients. As well as leprosy patients, household contacts of untreated patients, hospital patients and a randomly selected group of indigenous subjects were studied. A total of 253 subjects were included in the study. All gave verbal informed consent for the tests, the results of which were made available to the medical officers in charge of leprosy control in the area. The numbers of subjects in each group are shown in Table 1, together with their age and sex distribution. The ethnic origin of the subjects was similar in all of the study groups. Clinical examination of all the subjects was performed by IAC and local leprosy control teams. In addition, biopsies were taken from the lesions of 40 untreated and 15 treated leprosy patients using a $4 \mathrm{~mm}$ skin punch biopsy. ${ }^{7}$ Leprosy patients were classified on both clinical and histological grounds using the Ridley-Jopling classification: ${ }^{8,9}$ the numbers in each of the patient groups are shown in Table 2. Untreated patients were newly diagnosed and had not received any treatment prior to skin testing. The treatment status of the treated patient group varied from a few days to many years, but 38 out of the 53 treated patients $(72 \%)$ had received less than 6

Table 1. Numbers of subjects in each group with age and sex distribution

\begin{tabular}{|c|c|c|c|c|c|}
\hline \multirow[b]{2}{*}{ Group } & \multirow[b]{2}{*}{ No. } & \multirow{2}{*}{$\begin{array}{l}\text { Male:female } \\
\text { ratio }\end{array}$} & \multicolumn{2}{|c|}{ Age (years) } & \multirow[b]{2}{*}{$\%<15$ years } \\
\hline & & & Mean & SD & \\
\hline New patients & 52 & $1: 0 \cdot 86$ & $33 \cdot 1$ & $11 \cdot 9$ & $5 \cdot 8$ \\
\hline Treated patients & 53 & $1: 0 \cdot 20$ & $34 \cdot 1$ & $10 \cdot 5$ & 0 \\
\hline Household contacts & 78 & $1: 0.95$ & $23 \cdot 4$ & $15 \cdot 0$ & $35 \cdot 9$ \\
\hline Hospital contacts & 20 & $1: 0 \cdot 82$ & $35 \cdot 2$ & $11 \cdot 1$ & 0 \\
\hline Control group & 50 & $1: 0 \cdot 79$ & $24 \cdot 9$ & $8 \cdot 1$ & 0 \\
\hline Total & 253 & $1: 0.68$ & $28 \cdot 9$ & $12 \cdot 9$ & $12 \cdot 2$ \\
\hline
\end{tabular}


Table 2. Number of leprosy patients according to treatment status and Ridley-Jopling classification ${ }^{9}$

\begin{tabular}{lccccccc}
\hline & \multicolumn{7}{c}{ Number of patients } \\
\cline { 2 - 8 } Class & TT & BT & BB & BL & LL & Idt & Total \\
\hline New patients & 4 & 23 & 1 & 9 & 8 & 7 & 52 \\
Treated patients & 0 & 29 & 1 & 9 & 14 & 0 & 53 \\
\hline
\end{tabular}

months therapy. Recently treated patients were receiving multiple drug therapy (MDT, WHO regime), while many of those treated for longer than 2 years had been recently changed from dapsone monotherapy to MDT. Of the 53 treated patients, $44(83 \%)$ had some disability, while only 22 of the 52 untreated patients $(42 \%)$ were disabled. Three groups of healthy subjects were tested: household contacts of untreated leprosy patients, hospital workers, and a group of indigenous 'controls' with no household leprosy contacts. Since they live in an endemic area, most of these 'control' subjects will inevitably have had some exposure to $M$. leprae and were included for comparison with the household contacts who were more likely to have had recent exposure. The age and sex distribution of the groups is broadly comparable, although it should be noted that the household contact group contained a high proportion of subjects under 15 years old and that most of the treated patients examined were male.

\section{SKIN TESTING}

Skin tests were perf ormed using 2 reagents, PPD ( $1: 1000$ dilution, Evans Medical, Middlesex, UK) and RSTA (Batch CD19), a new tuberculin containing soluble protein antigens of armadilloderived $M$. leprae. ${ }^{5,10}$ In each subject, $0 \cdot 1 \mathrm{ml}$ of each reagent was injected intradermally on the volar aspect of opposing forearms. The injection site was ringed with a ball-point pen and the skin tests were read at $72 \mathrm{~h}$. Only 10 subjects $(4 \%)$ failed to return for skin test reading ( 2 household contacts, 2 hospital contacts, 1 new patient, and 5 'control' subjects). On 25 occasions it was necessary for 1 of 3 readers other than IAC to read the result. Measurement of positive responses was perf ormed using the 'ball pen' method developed by Sokal. ${ }^{11}$ This has the advantage that it is easily taught to other readers and has good reproducibility compared with other techniques. Results are expressed as average diameters: those less than $5 \mathrm{~mm}$ were taken as negative and the observations were analysed using a chi-squared test with Yates correction.

\section{Results}

The results for the paucibacillary patients, graded TT-BT or Idt by the Ridley-Jopling classification, are shown in Figure 1. There are significantly more negative reactions to PPD among the untreated patients than there are in the treated group $(p<0.02)$. Although there is a difference in the number of positive reactions to RSTA in the paucibacillary patients, this does not achieve statistical significance. If the indeterminate patients are removed from the paucibacillary group, the PPD results still show a statistically significant difference $(p<0.02)$ between the untreated $(57 \cdot 7 \%$ positive, $n=26)$ and treated paucibacillary patients $(89.7 \%$ positive, $n=29)$. Of the 7 indeterminate patients, 4 were PPD positive and 4 were RSTA positive.

The multibacillary patients (BB-LL) show many fewer positive reactions to PPD in the untreated group $(p<0.05)$ and their diameter is less than the positive responses shown by the treated multibacillary patients (Figure 2). The RSTA results show a virtually complete lack of 


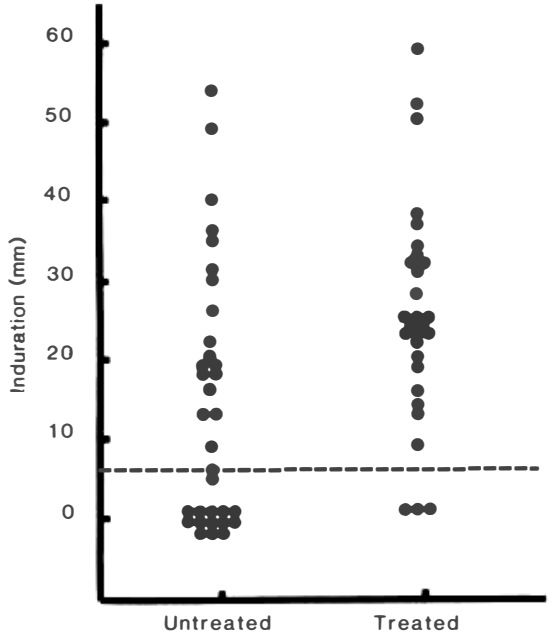

$\begin{array}{llll}\text { \% Positive } & 57.6 \quad 89.7 \quad(p<0.02)\end{array}$

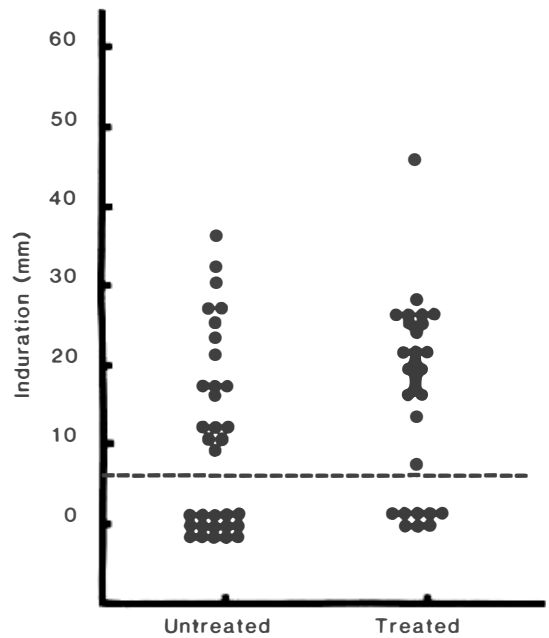

\% Positive $\quad 56.3$

72.4

(NS)

Figure 1. Skin test results for paucibacillary leprosy patients. The dotted line represents $5 \mathrm{~mm}$ induration, below which tests were regarded as negative.

PPD - Multibacillary Patients

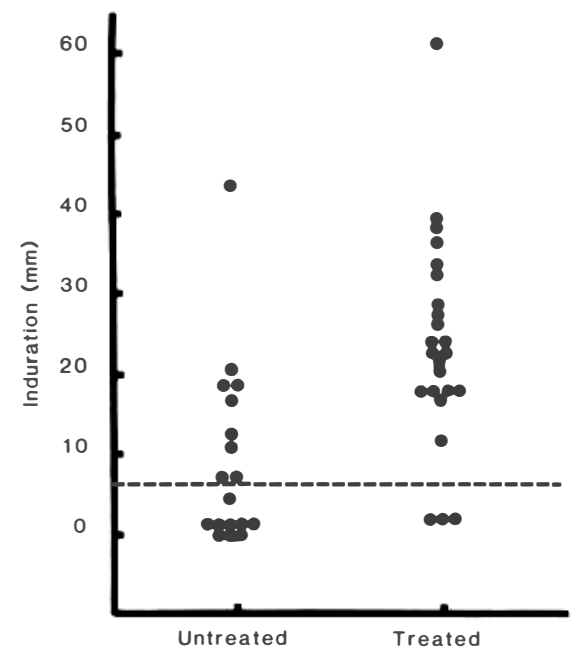

\% Positive

50.0

$87.5 \quad(p<0.05)$

\% Positive

11.1

$58.3 \quad(p<0.01)$

Figure 2. Skin test results for multibacillary leprosy patients. The dotted line represents $5 \mathrm{~mm}$ induration, below which tests were regraded as negative. 


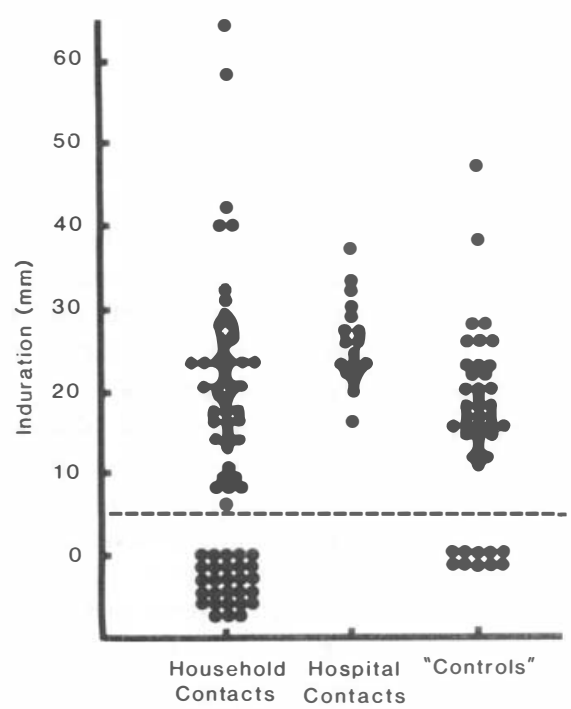

\% Positive

62.7

100.0

77.8

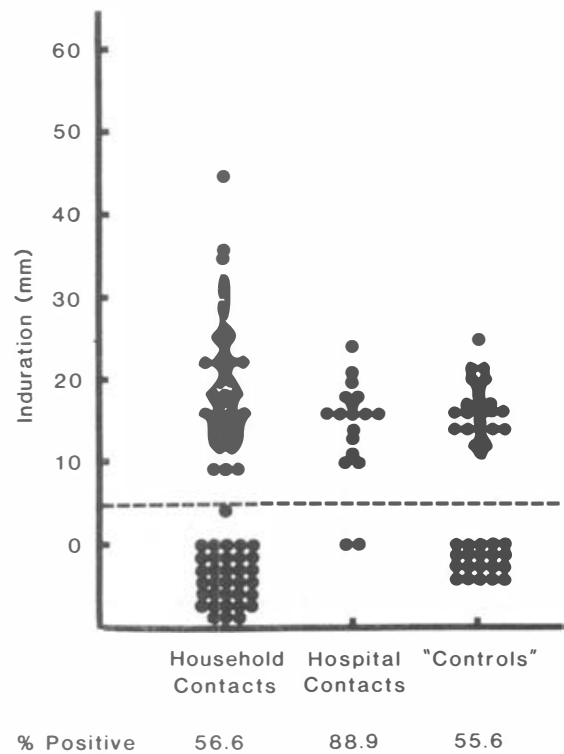

Figure 3. Skin test results for healthy subjects. The dotted line represents $5 \mathrm{~mm}$ induration, below which tests were regarded as negative.

reaction among the untreated multibacillary patients (only 2 positive), but more than half of the treated multibacillary patients react $(p<0 \cdot 01)$.

The results of the skin tests performed on the healthy subjects are shown in Figure 3. The number of responders to both PPD and RSTA are similar amongst the household contacts and the indigenous 'control' group. Although slightly fewer household contacts show positive responses to RSTA, this difference is not statistically significant. However, the diameter of the positive responses to RSTA amongst the household contacts is greater than those of the 'control' subjects. It was notable that all of the hospital contacts responded to PPD and that all but 2 responded to RSTA.

The household contacts and indigenous 'controls' showed a significantly higher response rate to RSTA than untreated multibacillary patients (both $p<0 \cdot 01$ ), but this difference disappears after treatment. There is no statistically significant difference in the response to RSTA between household contacts or indigenous 'controls' and paucibacillary patients. Although a smaller percentage of untreated multibacillary patients respond to PPD than either 'control' subjects or household contacts, this does not achieve statistical significance. Higher response rates to PPD are found in both paucibacillary and multibacillary treated patients than in household contacts ( $p<0.02$ and $p<0.05$ respectively), but there is no difference between the response of treated patients and household contacts to RSTA. Comparison of treated patients with the indigenous "control' subjects shows no statistical difference in their PPD or RSTA responsiveness, although the treated patients have a slightly higher response rate to PPD than the 'control' subjects.

Few side-effects were noted by the patients or healthy subjects, although several of the hospital contacts and one family contact complained of pyrexial reactions. No necrotic reactions were encountered, although several giant reactions greater than $40 \mathrm{~mm}$ average diameter occurred in response to both reagents. Some of the larger reactions were tender, but none required treatment and all had resolved in 1-2 weeks. 


\section{Discussion}

PPD contains large amounts of common mycobacterial antigen (groups i and ii) and some specific (group iv) antigen of M. tuberculosis. ${ }^{12}$ In contrast, new tuberculins contain less of the common antigens (groups i and ii) and are rich in specific antigen (group iv). Thus RSTA contains mainly specific $M$. leprae derived antigen. Paucibacillary patients (BT-TT) show good cell-mediated immunity to $M$. leprae in their lesions ${ }^{8}$ and they show strong responses in lymphocyte stimulation tests to M. leprae. ${ }^{2}$ Yet despite such responses, they still develop the disease and BT patients will often downgrade on the Ridley-Jopling scale if they are not treated. Such downgrading has previously been associated with decreased skin test reactivity to PPD. ${ }^{4}$ Our observations show that untreated paucibacillary patients have a decreased skin test response to both PPD and RSTA despite their apparently good intralesional immunity to $M$. leprae. This may reflect partial suppression of the effector mechanism of DTH to common mycobacterial antigens.

Consistent with this explanation, the percentage of paucibacillary patients with positive skin tests to RSTA is greater in the treated group, while the area of induration amongst the responders is not significantly different. Thus specific DTH to $M$. leprae appears to exist in both treated and untreated paucibacillary patients, and the difference between the two groups may reflect general suppression of DTH to mycobacteria in untreated patients. This contrasts with the multibacillary group in whom both the percentage of responders and the diameter of the positive responses is greater in the treated group, perhaps reflecting the emergence of specific DTH to M. leprae as well as increased DTH to common mycobacterial antigen. It should be noted that only 1 of the untreated patients was classified LLp on histology: the remainder were LLs or BL. In Indonesia, where most lepromatous patients are of polar type, RSTA has been shown to produce few positive responses in treated lepromatous patients. ${ }^{13}$ Our results suggest that RSTA skin tests can be positive in subpolar lepromatous patients, particularly if they have received treatment.

A large number of possible mechanisms have been suggested for the immune suppression which occurs in leprosy, particularly the lepromatous form. ${ }^{3}$ Many of the mechanisms postulated are not mutually exclusive and our data suggests that both general and specific suppression may coexist. Both abnormal antigen presentation and/or the production of suppressor lymphocytes could produce specific effects, while nonspecific suppression of DTH could be produced by humoral factors. Sequestration of antigen-reactive lymphocytes in lesions might also produce suppression of skin test reactions. The relative importance of these mechanisms at diff erent times during the course of the disease may also vary.

The healthy subjects included in this study show a number of interesting comparisons. The striking reactivity to both reagents shown by the hospital contacts probably reflects their constant exposure to mycobacteria, but these subjects also have a considerably better standard of living than the other groups. Similar enhanced responsiveness to RSTA and new tuberculin has been seen in healthy hospital workers in East Java. ${ }^{14,15}$

It is interesting to note the slightly diminished responsiveness of the household contacts compared with the indigenous 'control' groups. This does not reach statistical significance and may reflect the larger number of young subjects in the former group, but it may be that some of the household contacts, those most at risk of contracting leprosy, are immunologically compromised as suggested by Strickland. ${ }^{16}$ The small differences in PPD reactivity between the treated patients and the household contacts can also be explained by this mechanism and by differences in immunological experience of mycobacteria.

There was no obvious relationship between treatment duration and the diameter of PPD or RSTA responses: this may reflect the small number of patients in the study or the time scale of reversal of immune suppression. Dapsone has been shown to reduce the incidence of reversal reactions in leprosy patients, ${ }^{17}$ but the mechanism of action is not clear and the effect of MDT has not been documented. The situation is complicated by the lack of objective methods of diagnosing reversal reactions in leprosy, although muscle function testing is useful in detecting neuritis. ${ }^{18,19}$ 
Our observations suggest that treatment of leprosy patients with multiple drug therapy enhances DTH to both common and specific mycobacterial antigens. It is important that studies of immunity in leprosy patients take account of the possible eff ect of treatment on their results and give adequate details of the treatment status of their patients. We consider untreated patients to be preferable for most investigations of the pathogenesis of the immunological deficits in leprosy. Nevertheless, since many chemotherapeutic agents used in leprosy have effects on the immune system and cause a radical alteration in the bacillary load, complimentary studies of treated patients are necessary in order to plan optimal treatment regimes. We recognize that retrospective studies are difficult to interpret unambiguously and that there are some differences between the untreated and treated groups (e.g. disability status) which may have influenced our results. We therefore hope to perform a prospective study of skin testing with PPD and RSTA in leprosy patients over the next few years.

\section{Acknowledgments}

The work in Bangladesh was funded by the award of the Becton Dickinson Travelling Scholarship to IAC by the Royal College of Pathologists. IAC was supported by LEPRA. The authors are most grateful to Dr M S Islam, Dr I H Cochrane, Dr T Ali, Mr K Skillicorn, and Mr S Halda for their assistance in Bangladesh.

\section{References}

1 Bjune G, Barnetson RStC, Ridley DS, Kronvall G. Lymphocyte transformation test in leprosy; correlation of the response with inflammation of lesions. Clin exp Immunol, 1976; 25: 85-94.

2 Myrvang B, Godal T, Ridley DS, Froland SS, Song YK. Immune responsiveness to Mycobacterium leprae and other mycobacterial antigens throughout the clinical and histopathological spectrum of leprosy. Clin exp Immunol, 1973; 14: 541-53.

${ }^{3}$ Harboe M. The immunology of leprosy. In: Leprosy. Hastings RC (ed.). New York: Churchill Livingstone, 1985 , pp. 53-87.

${ }^{4}$ Guinto RS, Mabalay MC. A note on the tuberculin reaction in leprosy. Int J Lepr, 1962; 30: $278-83$.

5 Editorial. New tuberculins. Lancet, 1984; i: 199.

${ }^{6}$ Stanford JL, Rook GAW. Environmental mycobacteria and immunisation with BCG. In: Medical Microbiology. Easman CSF, Jeljaszewki J (eds), 1983, Vol. 2, pp 43-9, Academic Press, Hew York

7 Cree IA, McDougall AC, Coghill G, Beck JS. Quantitation of the granuloma fraction in leprosy skin biopsies by planimetry. Int $J$ Lepr, 1985; 53: 582-6.

${ }^{8}$ Ridley DS, Jopling WH. Classification of leprosy according to immunity: a five group system. Int J Lepr, 1966; 34: 255-73.

9 Ridley DS. Skin Biopsy in Leprosy, 2nd edn. Documenta Geigy, 1985.

10 Smelt AHM, Rees RJW, Liew FY. Induction of delayed-type hypersensitivity to Mycobacterium leprae in healthy individuals. Clin Exp Immunol, 1981; 44: 501-6.

11 Sokal JE. Measurement of delayed skin test responses. New Engl J Med, 1975; 293: 501-2.

12 Stanford JL, Grange JM. The meaning and structure of species as applied to mycobacteria. Tubercle, 1974; 55: $143-52$.

13 Smelt AHM, Rees RJW, Liew FY. Failure to induce delayed-type hypersensitivity to Mycobacterium leprae in long-term treated lepromatous leprosy patients. Clin Exp Immunol, 1981; 44: 507-11.

14 Grange JM, Beck JS, Harper EI, Kardjito T, Stanford JL. The effect of exposure of hospital employees to patients with tuberculosis on dermal reactivity to new tuberculin. Tubercle, 1986; 67: 109-18.

15 Morley SM, Beck JS, Brown RA, Grange JM, Kardjito T. Occupational exposure to Mycobacterium tuberculosis and cytology of response to skin testing with new tuberculin. Tubercle, 1987; 68: 71-3.

16 Strickland NH. The influence of immunosuppression and immunodeficiency on infections with leprosy and tuberculosis. Int J Lepr, 1985; 53: 86-100.

17 Barnetson RStC, Pearson JMH, Rees RJW. Evidence for prevention of borderline leprosy reactions by dapsone. Lancet, 1976; ii: 1171-2.

18 Duncan ME, Melsom R, Pearson JMH, Ridley DS. The association of pregnancy and leprosy. I. New cases, relapse of cured patients and deterioration of patients on treatment during pregnancy and lactation: results of a prospective study of 154 pregnancies in 147 Ethiopian women. Lepr Rev, 1981; 52: 245-62.

19 Duncan ME, Pearson JMH. Neuritis in pregnancy and lactation. Int J Le pr, 1982; 50: 31-8. 


\section{TDR; invitations to consider research projects in leprosy}

In the UNDP/World Bank/WHO Special Programme for Research and Training in Tropical Diseases 'TDR News' of Autumn 1987, Number 24, pages 6 and 7 carry invitations to scientists and doctors to participate in certain areas of research. Included in the 'six diseases', proposals for work in leprosy are listed as follows:

- development and validation of seroepidemiological methods, e.g. specific tests for antibody/antigen detection, for Mycobacterium leprae using molecular probes and for cell-mediated immune (CMI) responses both in vivo and in vitro;

- development of prophylactic vaccine(s): development of candidate subunit structures, establishment of effective vectors for immunization, identification of CMI-inducing antigens/epitopes, production of recombinant and/or synthetic vaccine candidates;

- identification of means to overcome pathogenic host responses: development of human and animal model systems, identification of genetic markers of disease, investigation of lymphocyte subsets and their repertoires and the role of lymphokines;

- identification of methods to prevent and control nerve damage: investigations of nerve damage in humans and animal models, determination of the role of CMI in nerve damage, identification of the function of cells/ antigens in tissue lesions;

- identification of means for better use of existing drugs: testing of new combinations in animal models, conduct of clinical trials in multibacillary patients using newer regimens;

- assessment of needs for improved therapy: study of relapses after cessation of treatment, identification of risk factors for relapse, survey of rif ampicin-resistant leprosy;

- identification of new drugs for leprosy: selection from inventory and/or design of new compounds, development of new microbial screens, conduct of animal studies for antileprosy activity, conduct of short-term clinical trials in humans;

- identification of new drugs for treatment of leprosy reactions: development of models for screening drug activity, testing of active drugs for pharmacological and mutagenic effects, toxicity and structure/activity relationships;

- evaluation of immunotherapeutic methods: conduct of immunoreactivity trials, study of immunotherapy combined with chemotherapy.

\section{Studies on the mode of action of, and resistance to antibiotics, Oxford, UK}

In the Oxford Medical SchoolGazette, Vol. XXXVIII, No 2, Michaelmas 1987, the Department of Microbiology and the Public Health Laboratory of the John Radcliffe Hospital, Oxford, report stated:

'A major determinant of how effective an antibiotic is against a particular bacterium is whether the antibiotic reaches its site of action within the cell sufficiently rapidly to halt growth. Drs Wright Nichols and Mary Slack are working on this topic. Current projects concern; how bacterial metabolism stimulates the uptake of aminoglycoside antibiotics, the interplay between beta-lactamases and outer membrane penetration of betalactam antibiotics (penicillins and cephalosporins) in Gram negative bacteria; and analysing quantitatively the diff usion of antibiotics into multicellular bacterial films or biofilms that can form on catheters and other medical implants.'

\section{Handbook of leprosy; W H Jopling; ELBS edition}

It is perhaps not fully appreciated that copies of this Handbook, published by William Heinemann Books Ltd, (22 Bedf ord Square, London WC1 B 3HH; Telephone 01-637-331), are available in an English Language Book Society edition at a price of only $£ 3.25$ sterling. Full inf ormation of the ELBS system was printed in this journal in March 1988, page 43. Order through the usual book trade channels either from the publisher or the publisher's agent or from such United Kingdom exporters as Abacus Books Ltd (Abacus House, Speldhurst Road, Tunbridge Wells, Kent TN4 0HU), Collet's Holdings Ltd (Denington Estate, Wellingborough, Northants NN8 2QT) or Gemini Book Distribution Ltd (Vale Road, Tonbridge, Kent TN9 1TD). When ordering please quote the International Standard Book Number (ISBN), which is 0433175680.

\section{Medical Education Newsletter, Dundee, Scotland}

This Newsletter from the Centre for Medical Education, University of Dundee, Dundee DD1 4HN, Scotland, UK, is available to bona fide applicants in the field of medical education, to whom it may well be invaluable. The most recent received in Oxf ord, Number 38, is in fact more than a newsletter in the usual sense of the word: it is an impressive document of 31 pages, full of practical information on: distance learning; educational technology; AIDS education; communication skills for medical students; assessing clinical competence and postgraduate education. The Centre also runs courses on various aspects of medical education throughout the year; further information from the above address. 